\title{
Cardiac MRI is safe in patients with pacemakers and defibrillators
}

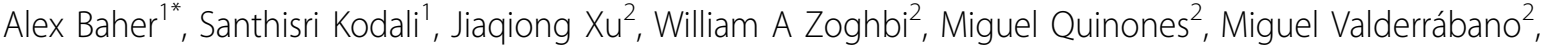 \\ Dipan J Shah
}

From 15th Annual SCMR Scientific Sessions

Orlando, FL, USA. 2-5 February 2012

\section{Background}

MRI has generally been contraindicated in patients (pts) with pacemakers (PM) or defibrillators (ICD) due to concern of a variety of potential complications. Several recent series have suggested potential safety of MRI in pts with cardiac devices, but most excluded cardiac or thoracic imaging. Additionally there is very limited data on safety of CMR in pts with devices, especially in pts with ICD.

\section{Methods}

Pts with devices underwent CMR on a 1.5-T Siemens Avanto scanner utilizing a pre-specified protocol that involved device interrogation and programming prior to, and immediately after CMR to assess lead impedance, battery voltage, and pacing capture/sensing thresholds. Devices in PM dependent pts (intrinsic HR $<60 \mathrm{bpm}$ ) were reprogrammed to asynchronous pacing mode, whereas devices in non-PM dependent pts were reprogrammed to pacing function deactivated. All underwent rhythm, pulse oximetry, and visual monitoring during CMR. All pts were scheduled for follow up device interrogation to evaluate for later changes in device parameters. For hospitalized pts troponin levels were measured prior to and 8 hours after CMR.

\section{Results}

Seventy nine CMR exams were performed in 64 pts [26 men, age $64.7 \pm 14.2$ years, 34 with $P M$, and 30 with ICD]. Of these, 47 exams [24 with PM, and 23 with ICD] were on inpatients who had troponin levels drawn before and after CMR. All pts underwent CMR without complications, specifically none complained of heating,

IInternal Medicine, The Methodist Hospital, Houston, TX, USA

Full list of author information is available at the end of the article and there were no malignant arrhythmias or device malfunctions noted. Follow up device interrogation was performed after $68(86 \%)$ of the exams at $13.2 \pm 20.8$ days after CMR. No device complications were noted during the follow up period. Table 1 and Table 2 summarize the changes in device parameters. We detected no significant rise in troponin levels with CMR $(0.046 \pm 0.200$ and $0.037 \pm 0.132, \mathrm{p}=0.596$ for pts with $\mathrm{PM}$; and value

\begin{tabular}{|l|c|c|c|c|c|c|}
\hline & \multicolumn{9}{|c|}{ Pacemaker } & \multicolumn{3}{c|}{ ICD } \\
\cline { 2 - 7 } & Pre-test & Post-test & $p$-value & Pre-test & Post-test & $p$-value \\
\hline LI Atrium & $445 \pm 96$ & $444 \pm 96$ & 0.7269 & $490 \pm 108$ & $491 \pm 108$ & 0.7289 \\
\hline LIVentricle & $478 \pm 92$ & $476 \pm 93$ & 0.1465 & $509 \pm 153$ & $503 \pm 148$ & 0.1030 \\
\hline Battery Voltage & $2.79 \pm$ & $2.79 \pm$ & 0.3242 & $3.03 \pm 0.21$ & $3.02 \pm 0.70$ & $0.0010^{\circ}$ \\
& 0.11 & 0.11 & & & & \\
\hline Atrial Capture & $0.51 \pm$ & $0.52 \pm$ & 0.1846 & $0.44 \pm 0.15$ & $0.51 \pm 0.25$ & 0.1425 \\
& 0.13 & 0.13 & & & & \\
\hline Ventricular & $0.46 \pm$ & $0.51 \pm$ & $0.0192^{*}$ & $0.44 \pm 0.22$ & $0.45 \pm 0.28$ & 0.6914 \\
Capture & 0.12 & 0.17 & & & & \\
\hline Atrial Sense & $1.98 \pm$ & $2.28 \pm$ & 0.0885 & $2.70 \pm 1.75$ & $2.58 \pm 1.67$ & 0.1078 \\
& 1.57 & 1.82 & & & & \\
\hline Ventricular Sense & $9.2 \pm 4.7$ & $9.3 \pm 4.7$ & $0.0139^{*}$ & $12.3 \pm 6.6$ & $12.0 \pm 6.5$ & 0.2170 \\
\hline
\end{tabular}

Figure 1 Device parameters for PM and ICD measures before and immediately after CMR.

\begin{tabular}{|l|c|c|c|c|c|c|}
\hline \multicolumn{9}{|c|}{} & \multicolumn{3}{|c|}{ Pacemaker } & \multicolumn{3}{c|}{ ICD } \\
\cline { 2 - 7 } & Pre-test & Follow up & $p$-value & Pre-test & Follow up & $p$-value \\
\hline L Atrium & $435 \pm 105$ & $426 \pm 109$ & 0.0609 & $479 \pm 96$ & $442 \pm 89$ & 0.0785 \\
\hline LIVentricle & $465 \pm 95$ & $478 \pm 126$ & 0.4758 & $476 \pm 90$ & $466 \pm 90$ & 0.2036 \\
\hline Battery Voltage & $2.80 \pm$ & $2.80 \pm$ & 0.1744 & $3.02 \pm 0.22$ & $3.01 \pm 0.22$ & $0.0187^{\circ}$ \\
& 0.12 & 0.11 & & & & \\
\hline Atrial Capture & $0.52 \pm$ & $0.70 \pm$ & $0.0004^{\circ}$ & $0.43 \pm 0.52$ & $0.51 \pm 0.27$ & 0.1644 \\
& 0.14 & 0.19 & & & & \\
\hline Ventricular & $0.47 \pm$ & $0.75 \pm$ & $0.0007^{*}$ & $0.48 \pm 0.25$ & $0.53 \pm 0.28$ & 0.2973 \\
Capture & 0.15 & 0.32 & & & & \\
\hline Atrial Sense & $1.76 \pm$ & $1.97 \pm$ & 0.4206 & $2.63 \pm 1.60$ & $2.29 \pm 1.60$ & $0.0023^{\circ}$ \\
& 1.26 & 1.41 & & & & \\
\hline Ventricular Sense & $9.58 \pm$ & $9.02 \pm$ & 0.1380 & $12.36 \pm$ & $11.42 \pm$ & 0.0517 \\
& 4.59 & 4.01 & & 6.75 & 6.32 & \\
\hline
\end{tabular}

Figure 2 Device parameters for PM and ICD measures at follow up compared with the pre-test values. 
$0.099 \pm 0.263$ and $0.108 \pm 0.247, \mathrm{p}=0.434$ for pts with ICD).

\section{Conclusions}

Patients with PM or ICD may safely undergo CMR using a pre-designed protocol without any clinically meaningful change in device function.

\section{Funding}

None.

\section{Author details}

${ }^{1}$ Internal Medicine, The Methodist Hospital, Houston, TX, USA. ${ }^{2}$ Methodist

DeBakey Heart \& Vascular Center, The Methodist Hospital, Houston, TX, USA.

Published: 1 February 2012

doi:10.1186/1532-429X-14-S1-0102

Cite this article as: Baher et al:: Cardiac MRI is safe in patients with

pacemakers and defibrillators. Journal of Cardiovascular Magnetic

Resonance 2012 14(Suppl 1):0102

Submit your next manuscript to BioMed Central and take full advantage of:

- Convenient online submission

- Thorough peer review

- No space constraints or color figure charges

- Immediate publication on acceptance

- Inclusion in PubMed, CAS, Scopus and Google Scholar

- Research which is freely available for redistribution

Submit your manuscript at www.biomedcentral.com/submit 